Original Paper http://ajol.info/index.php/ijbcs http://indexmedicus.afro.who.int

\title{
Inventaire et distribution des principaux arthropodes ravageurs du manioc (Manihot esculenta CRANTZ) au Tchad
}

\author{
Mahamat-Saleh ABDERAHIM ${ }^{1,2^{*}}$, Mamadou DIATTE ${ }^{1}$, Babacar LABOU ${ }^{1}$, \\ Gallo SOW $^{1}$ et Karamoko DIARRA ${ }^{1}$
}

\footnotetext{
${ }^{1}$ Université Cheikh Anta Diop de Dakar, Laboratoire de Production et Protection Intégrées en Agro écosystèmes- L2PIA, Faculté des Sciences et Techniques, Dakar, Sénégal.

${ }^{2}$ Université Adam Barka d'Abéché du Tchad, Département de Biologie, Faculté des Sciences et Techniques.

*Auteur correspondant ; E-mail : mahamatsaleh96@yahoo.fr; Tel : +23566289501; +221 785256929
}

\section{REMERCIEMENTS}

Nous remercions profondément la Commission Nationale de Formation des Formateurs (CONFOFOR) du Tchad pour le financement de cette étude.

\section{RESUME}

Le manioc joue un rôle important dans l'alimentation des populations. Cependant les ravageurs constituent une contrainte à sa production. Dans le but d'identifier les arthropodes ravageurs du manioc $(M$. esculenta) et d'étudier leur distribution, une étude a été réalisée au Tchad. Les échantillonnages ont été effectués dans les sites de Kobdogue, Moya, Faya et Yaroungou. Tous les ravageurs rencontrés ont été récoltés et identifiés. Huit espèces de ravageurs ont été répertoriées: Phenacoccus manihoti, Bemisia tabaci, Mononychellus tanajoa, Zonocerus variegatus, Odontotermes sp aff erraticus, Aromia moschata, Camponotus ligniperda et Panonychus ulmi. La cochenille farineuse ( $P$. manihoti) a été observée dans tous les sites. Elle est plus importante à Faya $(50,76 \%)$ et à Kobdogue $(31,64 \%)$. L'acarien vert $M$. tanajoa et la mouche blanche $B$. tabaci, sont respectivement plus importants à Kobdogue (50,63\% et 39,17\%) et à Faya $(18,98 \%$ et 24,62\%). La variété Ngaloum nguegdje est la plus attaquée par les ravageurs : la cochenille farineuse ( $\mathrm{n}=14087)$, l'acarien vert $(\mathrm{n}=524)$ et la mouche blanche $(\mathrm{n}=3102)$ comparée à la variété Kangaba respectivement $(\mathrm{n}=3042),(\mathrm{n}=$ $229)$ et $(n=1675)$. Ces résultats permettront d'adapter une stratégie de gestion des arthropodes ravageurs du manioc au Tchad.

(C) 2018 International Formulae Group. All rights reserved.

Mots clés : Manihot esculenta, bio-agresseurs, Phenacoccus manihoti, Mononychellus tanajoa, Bemisia tabaci, Afrique centrale. 


\title{
Inventory and distribution of the main arthropod pests of cassava (Manihot esculenta CRANTZ) in Chad
}

\begin{abstract}
Cassava plays an important role in the diet of the population. However, pests are a constraint to its production. In order to identify arthropod pests of cassava (M. esculenta) and study their distribution, a study was carried out in Chad. Sampling was carried out at the sites of Kobdogue, Moya, Faya and Yaroungou. All pests encountered were harvested and identified. Eight pest species have been identified: Phenacoccus manihoti, Bemisia tabaci, Mononychellus tanajoa, Zonocerus variegatus, Odontotermes sp aff erraticus, Aromia moschata, Camponotus ligniperda and Panonychus ulmi. The mealybug (P. manihoti) was observed at all sites. It is higher in Faya (50.76\%) and Kobdogue (31.64\%). The green mite M. tanajoa and the white fly B. tabaci are more important in Kobdogue (50.63\% and $39.17 \%$ respectively) and Faya (18.98\% and $24.62 \%)$. The variety Ngaloum nguegdje is the most attacked by pests: mealybug $(\mathrm{n}=14087)$, green mite $(\mathrm{n}=$ $524)$ and white fly $(\mathrm{n}=3102)$ compared to the variety Kangaba respectively $(\mathrm{n}=3042),(\mathrm{n}=229)$ and $(n=1675)$. These results will make it possible to adapt a strategy for the management of arthropod pests of cassava in Chad.

(c) 2018 International Formulae Group. All rights reserved.
\end{abstract}

Keywords : Manihot esculenta, bio-aggressors, Phenacoccus manihoti, Mononychellus tanajoa, Bemisia tabaci, Central Africa.

\section{INTRODUCTION}

Le manioc est l'une des tubercules les plus consommées en Afrique de l'ouest et central. Il peut contribuer à résorber les problèmes de carence en vitamine $A$ (Djinadou et al., 2018). Au Tchad, la culture du manioc joue un rôle déterminant dans la stratégie de la sécurité alimentaire. Environ, 50000 ha sont consacrés chaque année à cette culture (FAO, 2012). Dans le Sud du Tchad, le manioc est plus souvent cultivé en association avec d'autres plantes (Nadjiam et al., 2016). Il constitue une denrée alternative aux déficits alimentaires de plus en plus récurrents ces dernières années et représente une source de diversification des revenus pour des petits exploitants agricoles.

Néanmoins, sa production est confrontée à plusieurs contraintes biotiques. Plusieurs pathogènes attaquent le manioc dont les maladies bactériennes et virales (Cacai et al., 2012). Il s'y ajoute les arthropodes ravageurs qui représentent les principaux obstacles biologiques à la culture du manioc (Mahamat Saleh, 2010).

Plusieurs ravageurs ont été recensés sur le manioc. Ils s'agissent de l'acarien vert, de la cochenille farineuse, du criquet, de la mouche blanche, des thrips et des punaises (Ntawuruhunga et al., 2002 ; Papy et Willy, 2012; Mahungu et al., 2014). L'acarien vert et la cochenille farineuse, ont été introduits accidentellement en Afrique et ont causé des dégâts économiques ou des pertes de rendement considérables sur cette culture (Bellotti et al., 2012). La mouche blanche $B$. tabaci, vecteur de la mosaïque du manioc, est un ravageur majeur du manioc en Afrique, causant de graves pertes de récolte (Calvert et Thresh, 2002). La multiplication végétative du manioc facilite le mouvement des arthropodes ravageurs d'un pays à un autre (Agre et al., 2015). Au Congo Brazzaville et au Congo Kinshasa, l'acarien vert, la cochenille farineuse, le criquet, la mouche blanche, les thrips et les punaises ont été répertoriés sur le manioc (Ntawuruhunga et al., 2002; 
Mahungu et al., 2014). Au Sénégal, les travaux de Faye (2016) ont également montré la présence de la cochenille farineuse, du criquet, de l'acarien vert et des termites. Il ressort de ses études que le type de variété de manioc influe sur les termites.

Aucune étude sur les arthropodes ravageurs du manioc n'a été jusque-là menée au Tchad. L'objectif de ce travail est de : (i) identifier les arthropodes ravageurs associés à la culture du manioc au Tchad et (ii) étudier leurs distributions en fonction des sites d'étude et des variétés cultivées.

\section{MATERIEL ET METHODES Sites d'étude}

La zone d'étude est située dans la partie soudanienne du Tchad où les conditions agro-climatiques sont favorables à la culture des plantes à racines et à tubercules. Les échantillonnages ont été effectués dans le département de la Grande Sido (région du Moyen chari) dans les sites de Kobdogue, Moya, Faya et Yaroungou (Figure 1) en milieu paysan. Le département de la grande Sido est situé à l'extrême sud du Tchad juste à $20 \mathrm{~km}$ de la frontière tchado-centrafricaine. Il est bordé à l'Ouest par le département de Barh Koh et à l'Est par le département de lac Iro. Son relief se caractérise par une savane parsemée de grands arbres fruitiers tels que les nérés, les karités, etc. La latérite donne au paysage une couleur rouge qui s'accentue progressivement. La végétation est une forêt claire avec des espèces variées et des sols riches et diversifiés parfois inondés et la faune est assez riche. Le climat est de type tropical, et la pluviométrie varie de 800 à $1200 \mathrm{~mm}$ par an.

\section{Culture de manioc}

L'étude a été réalisée en milieu paysan dans les villages de Kobdogue, Moya, Faya et Yaroungou en saison sèche. Dans chaque village, 5 champs de manioc ont été choisis. Chaque champ est constitué de 100 lignes espacées de $1 \mathrm{~m}$. Sur chaque ligne, 100 plants distants de $1 \mathrm{~m}$ ont été repiqués. Au total, 10000 plants contenus dans 1 ha ont été obtenus. Le bouturage est la technique de multiplication utilisée par les producteurs. Aucun traitement phytosanitaire n'a été utilisé durant la période de culture. Un sarclage a été effectué avant et après le repiquage des boutures. Les variétés de manioc utilisées ont été obtenues à la suite d'une enquête monographique et des observations faites sur le terrain.

\section{Echantillonnage des ravageurs}

Des comptages mensuels ont été effectués pour quantifier l'abondance des ravageurs sur le terrain. Vingt champs ont été prospectés à raison de 5 par village. Un échantillon de 30 pieds de manioc a été prospecté au hasard par champs. Sur chaque pied, 3 rejets ont été aléatoirement choisis à la base. Pour chaque rejet, 9 feuilles ont été choisies du bas vers le haut: vieilles feuilles (3 dernières feuilles du bas), jeunes feuilles ( 3 dernières feuilles avant l'apex) et feuilles apicales ( 3 feuilles du haut). Au total, 810 feuilles ont été tirées au hasard et examinées par champ. Le comptage des ravageurs rencontrés a été effectué en mentionnant les stades immatures et les adultes.

Les ravageurs échantillonnés sont conservés dans l'alcool à $70^{\circ}$. Ils ont été ensuite identifiés à l'aide de la collection de référence du laboratoire de production et protection intégrées des Agroécosystèmes de la Faculté des Sciences et Techniques de l'Université Cheikh Anta Diop de Dakar (UCAD).

\section{Analyses statistiques}

Les données normalisées ont été soumises à une analyse de variance (ANOVA). L'abondance des stades de développement (stade immature et stade mature) des espèces de ravageurs selon les variétés de manioc et les sites d'études a été analysée à l'aide d'une ANOVA avec le logiciel $\mathrm{R}$ version i3863.2.3. Les moyennes ont été séparées par le test de StudentNewman keuls (SNK). Le niveau de signification est maintenu à $5 \%$ pour toutes les analyses. 


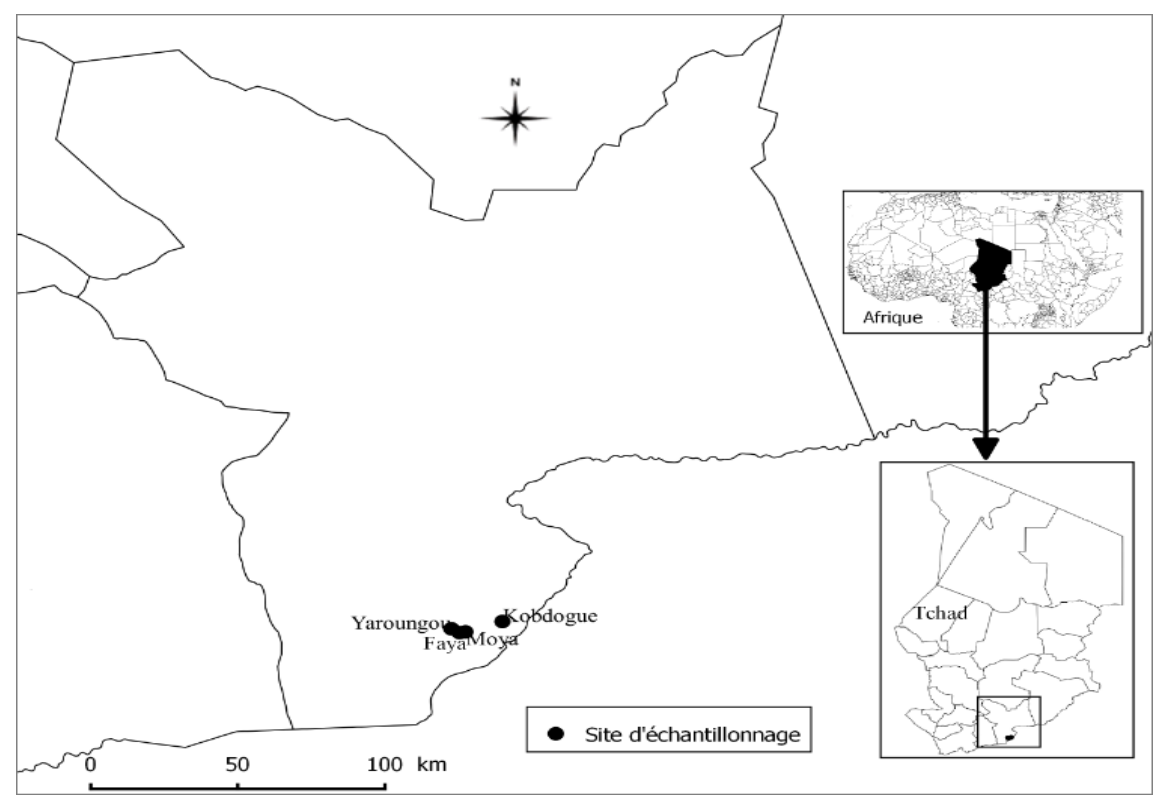

Figure 1 : Localisation des sites d'étude.

\section{RESULTATS}

\section{Identification des arthropodes ravageurs} associés aux variétés locales du manioc

Différentes espèces d'arthropodes ont été identifiées sur les deux variétés (Nagaloum ngeugdje et Kangaba) de manioc. Les huit espèces identifiées sont réparties dans 7 ordres et 9 familles. L'espèce $P$. manihoti a été la plus abondante sur les deux variétés, suivie de B. tabaci et de M. tanajoa (Tableau 1). Outre ces principaux ravageurs, d'autres bio-agresseurs appartenant aux ordres des orthoptères (Zonocerus variegatus), des isoptères (Odontotermes sp aff erraticus), des Hyménoptères (Camponotus ligniperda) des coléoptères (Aromia moschata) et des Arachnides (Panonychus ulmi) ont été aussi récoltées (Tableau 1).

Distribution des populations de la cochenille farineuse ( $P$. manihoti) en fonction des sites et des variétés cultivées

L'abondance de $P$. manihoti a montré une variabilité significative en fonction des sites. Le nombre des stades immatures de $P$. manihoti a été plus important dans le site de Faya suivi de Kobdogue, de Moya et de
Yaroungou $(\mathrm{ddl}=3 ; \mathrm{F}=36,04 ; \mathrm{P}<0,0001)$. La même tendance a été observée au niveau des stades matures qui sont également plus abondants dans les sites de Faya et celui de Kobdogue que dans les sites de Moya et de Yaroungou $(\mathrm{ddl}=3 ; \mathrm{F}=32,51 ; \mathrm{P}<0,0001)$ (Figure 2).

La variété de manioc influe sur les stades immatures et les stades matures de $P$. manihoti $(\mathrm{ddl}=1 ; \mathrm{F}=32,292 ; \mathrm{P}=0,0002)$ et ( $\mathrm{ddl}=1 ; \mathrm{F}=49,93 ; \mathrm{P}<0,0001)$. Le nombre d'individus des deux stades est plus élevé sur la variété Ngaloum nguegdje comparée à la variété Kangaba (Figure 3).

Distribution des populations de l'acarien vert (M. tanajoa) en fonction des sites et des variétés cultivées

Le nombre d'individus de l'espèce M. tanajoa est significativement différent en fonction de sites $(\mathrm{ddl}=3 ; \mathrm{F}=69,19 ; \mathrm{P}<$ $0,0001),(\mathrm{ddl}=3 ; \mathrm{F}=36,04 ; \mathrm{P}<0,0001)$. Le nombre d'individus des stades immatures et des stades matures de l'acarien vert est plus important dans le site de Kobdogue suivi de Faya et Moya et plus faible dans le site de Yaroungou (Figure 4). 
L'abondance de l'acarien vert a montré une variabilité significative en fonction des variétés $(\mathrm{ddl}=1 ; \mathrm{F}=95,25 ; \mathrm{P}<$ $0,0001),(\mathrm{ddl}=1 ; \mathrm{F}=95,25 ; \mathrm{P}<0,0001) . \mathrm{La}$ variété Ngaloum nguegdje abrite plus d'acarien (stade immature et stade mature) que la variété Kangaba (Figure 5).

\section{Distribution des populations de la mouche blanche (Bemisia tabaci) en fonction des sites et des variétés cultivées}

Le site influe sur l'abondance des différents stades de $B$. tabaci $(\mathrm{ddl}=3 ; \mathrm{F}=$
$0,735 ; \mathrm{P}=0,672)$ et $(\mathrm{ddl}=3 ; 0,85 ; \mathrm{P}=$ $0,64)$. Le nombre d'individus de la mouche blanche tout stade confondu est plus important sur le site de Kobdogue suivi de Faya. Les plus faibles nombres sont observés dans les sites de Moya et de Yaroungou (Figure 6).

La variété Ngaloum nguegdje est plus infestée par les stades de la mouche blanche que la variété Kangaba $(\mathrm{ddl}=1 ; \mathrm{F}=$ $15,88 ; \mathrm{P}=0,0025) ;(\mathrm{ddl}=1 ; \mathrm{F}=7,016 ; \mathrm{P}=$ $0,025)$ (Figure 7).

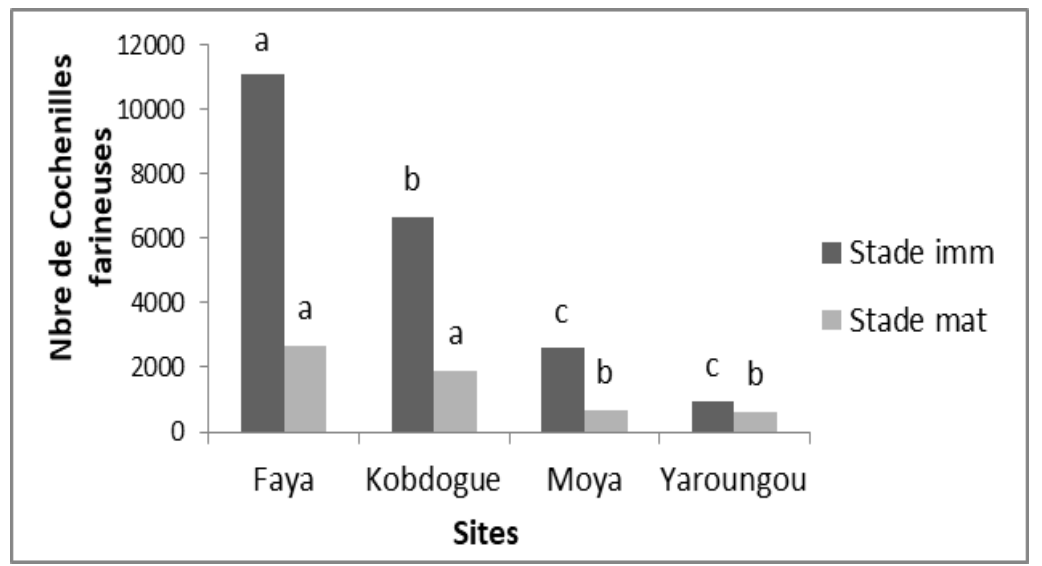

Figure 2 : Abondance des stades de développement de la cochenille farineuse $P$. manihoti en fonction des sites. Nbre $=$ Nombre, Stade $i m m=$ Stade larvaire, Stade mat $=$ stade adulte .

Les histogrammes ayant les mêmes lettres ne sont pas significativement différentes au seuil de 5\%, test SNK.

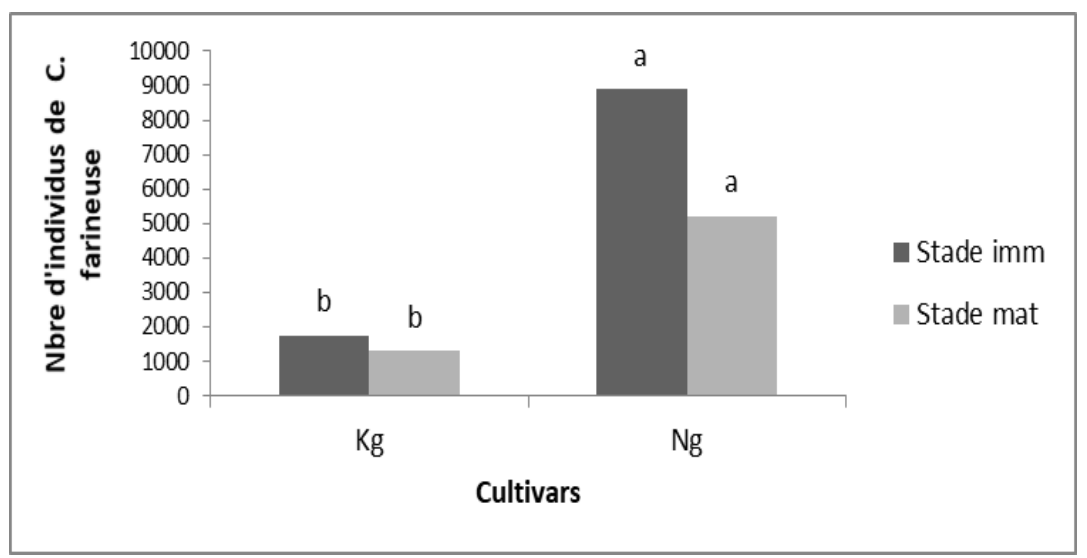

Figure 3 : Taux d'infestation de la cochenille farineuse en fonction des cultivars. $(\mathrm{Ng})=$ Ngaloum nguegdje, $(\mathrm{Kg})=$ Kangaba $; \mathrm{Nbre}=$ Nombre $;$ Stade $\mathrm{imm}=$ Stade larvaire, Stade mat $=$ stade adulte . Les histogrammes ayant les mêmes lettres ne sont pas significativement différentes au seuil de 5\%, test SNK. 


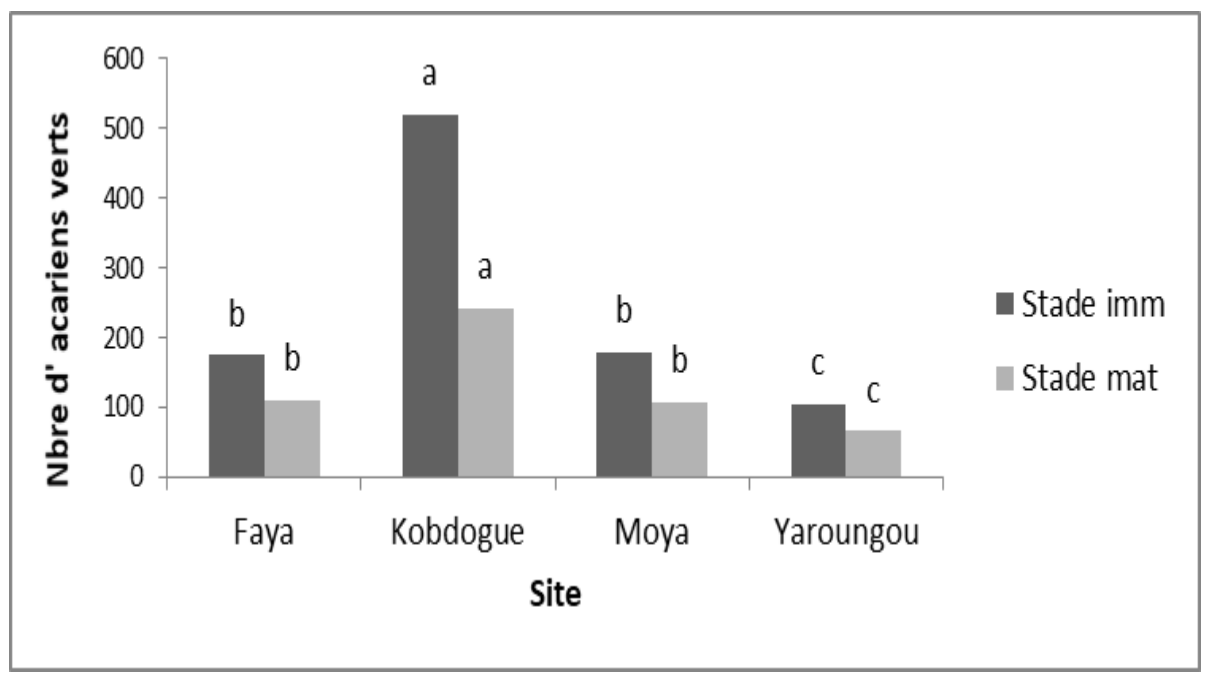

Figure 4 : Abondance des stades de développement de l'acarien vert M. tanajoa en fonction des sites. Stade imm= Stade larvaire, Stade mat=stade adulte. Les histogrammes ayant les mêmes lettres ne sont pas significativement différentes au seuil de 5\%, test SNK.

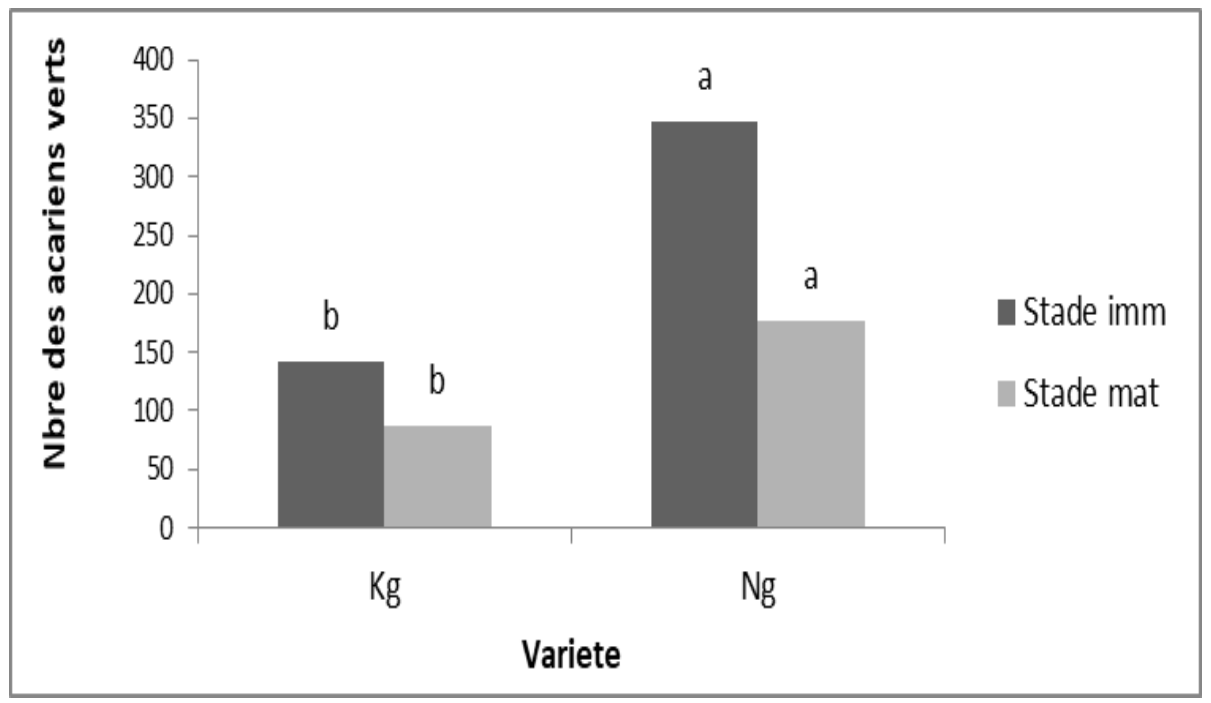

Figure 5 : Abondance des stades de développement de l'acarien vert Mononychellus tanajoa en fonction des variétés.

$(\mathrm{Ng})=$ Ngaloum nguegdje, $(\mathrm{Kg})=$ Kangaba $;$ Stade imm $=$ Stade larvaire, Stade mat=stade adulte. Les histogrammes ayant les mêmes lettres ne sont pas significativement différentes au seuil de 5\%, test SNK 


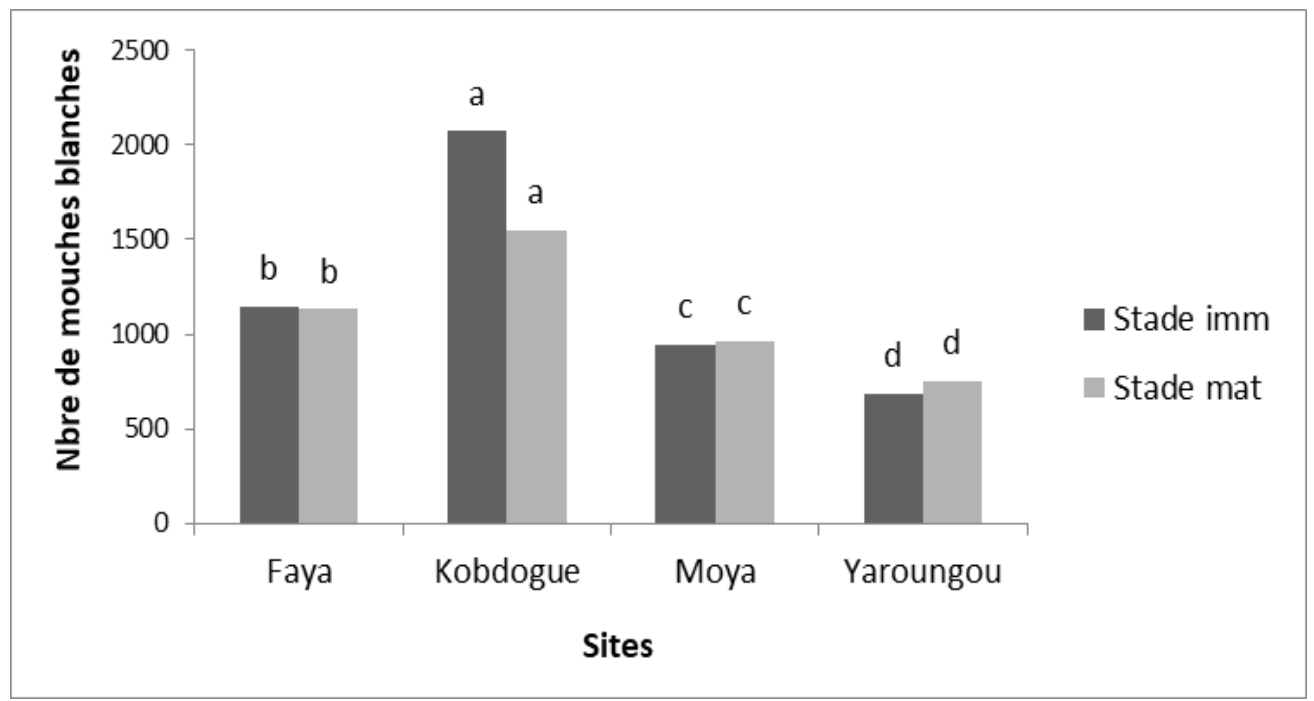

Figure 6 : Abondance des stades de développement de la mouche blanche Bemisia tabaci en fonction des sites.

Stade $\mathrm{imm}=$ Stade larvaire, Stade mat=stade adulte. Les histogrammes ayant les mêmes lettres ne sont pas significativement différentes au seuil de $5 \%$, test SNK.

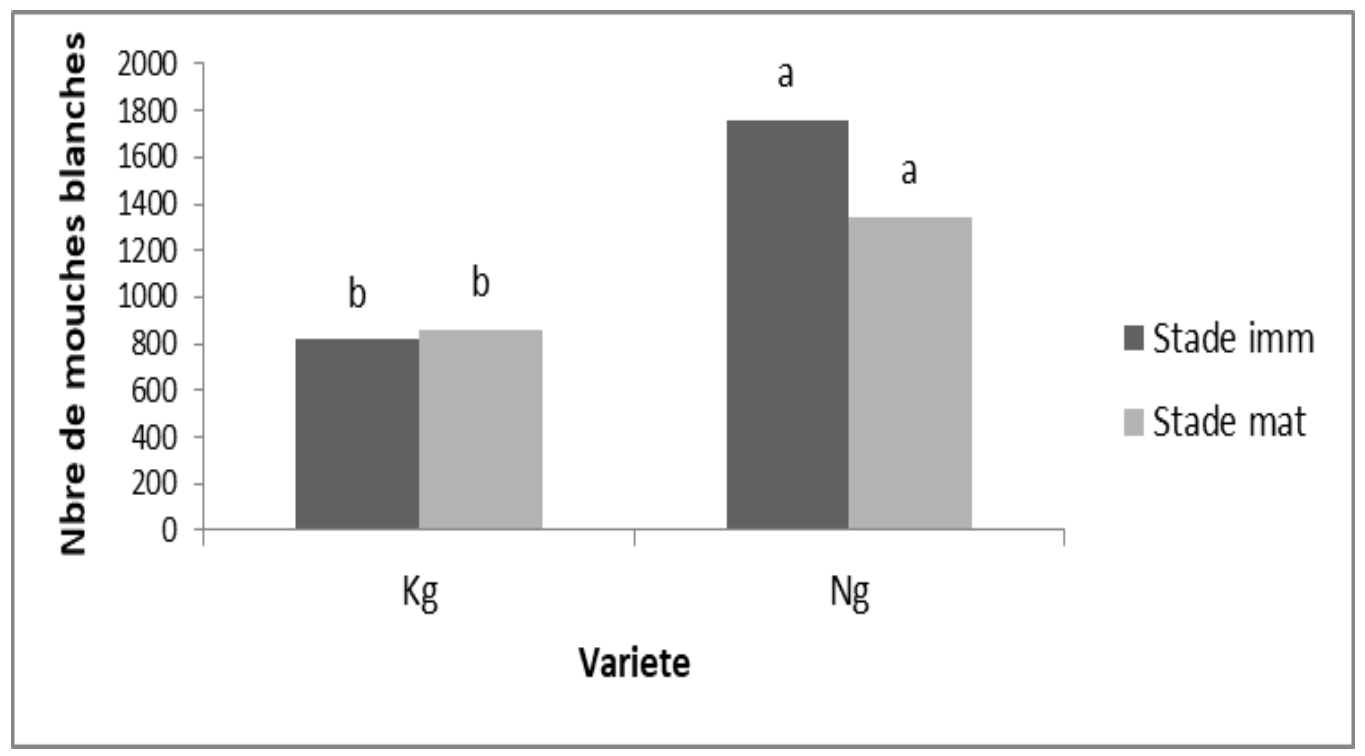

Figure 7 : Abondance de Bemisia tabaci en fonction des variétés. (Ng)=Ngaloum nguegdje, $(\mathrm{Kg})=$ Kangaba $;$ Stade $\mathrm{imm}=$ Stade larvaire, Stade mat=stade adulte. Les histogrammes ayant les mêmes lettres ne sont pas significativement différentes au seuil de $5 \%$, test SNK. 
Tableau 1 : Arthropodes ravageurs associés à la culture du manioc.

\begin{tabular}{|c|c|c|c|}
\hline \multirow{3}{*}{$\begin{array}{l}\text { Ordre } \\
\text { Famille } \\
\text { Espèce }\end{array}$} & \multicolumn{2}{|c|}{ Nombre d'individus récoltés (\% du total) } & \multirow{3}{*}{ Statut trophique } \\
\hline & \multicolumn{2}{|c|}{ Variétés } & \\
\hline & Ngaloum nguegdje & Kangaba & \\
\hline Orthoptère & \multirow{3}{*}{$112(0,24)$} & \multirow{3}{*}{$99(0,58)$} & \multirow{3}{*}{ Ravageur } \\
\hline Pyrgomorphidae & & & \\
\hline Zonocerus variegatus & & & \\
\hline Homoptère & \multirow{3}{*}{$34495(72,90)$} & \multirow{3}{*}{$9655(56,38)$} & \multirow{3}{*}{ Ravageur } \\
\hline Pseudococcidae & & & \\
\hline Phenacoccus manihoti & & & \\
\hline Coléoptère & & \multirow{3}{*}{$41(0,24)$} & \multirow{3}{*}{ Ravageur } \\
\hline Cerambycidae & \multirow{2}{*}{$65(0,14)$} & & \\
\hline Aromia moschata & & & \\
\hline Hyménoptère & & \multirow{3}{*}{$43(0,25)$} & \multirow{3}{*}{ Ravageur } \\
\hline Formicidae & \multirow{2}{*}{$61(0,13)$} & & \\
\hline Camponotus ligniperda & & & \\
\hline Arachnide & & \multirow{3}{*}{$172(1,00)$} & \multirow{3}{*}{ Ravageur } \\
\hline Tetranychidae & \multirow{2}{*}{$328(0,69)$} & & \\
\hline Mononychellus tanajoa & & & \\
\hline Hémiptère & \multirow{3}{*}{$12031(25,43)$} & \multirow{3}{*}{$7028(41,04)$} & \multirow{3}{*}{ Ravageur } \\
\hline Aleyrodidae & & & \\
\hline Bemisia tabaci & & & \\
\hline Arachnide & & \multirow{3}{*}{$12(0,07)$} & \multirow{3}{*}{ Ravageur } \\
\hline Tetranychidae & \multirow{2}{*}{$32(0,07)$} & & \\
\hline Panonychus ulmi & & & \\
\hline Isoptère & & \multirow{3}{*}{$38(0,22)$} & \multirow{4}{*}{ Ravageur } \\
\hline Termitidea & \multirow[b]{2}{*}{$87(0,18)$} & & \\
\hline $\begin{array}{l}\text { Odontotermessp aff } \\
\text { erraticus }\end{array}$ & & & \\
\hline Total des récoltes & 47211(100\%) & $17088(100 \%)$ & \\
\hline
\end{tabular}

\section{DISCUSSION}

Le manioc est attaqué par une diversité importante d'espèces d'arthropodes dominées par la cochenille farineuse $P$. manihoti, la mouche blanche $B$. tabaci et l'acarien vert $M$. tanajoa. Au regard des résultats obtenus, ces espèces constituent les principaux ravageurs du manioc pour cette étude dans cette région du Tchad. Ces résultats confirment ceux de l'Institut International d'Agriculture Tropicale [IITA] (2000). Outre ces principaux ravageurs, d'autres bio-agresseurs appartenant aux ordres des orthoptères (Zonocerus variegatus), des isoptères (Odontotermes sp aff erraticus), des Hyménoptères (Camponotus ligniperda) des coléoptères (Aromia moschata) et des Arachnides (Panonychus ulmi) attaquent aussi le manioc. Peu d'espèces ont été recensées par Mahungu et al. (2014) en République Démocratique du Congo. Les espèces Odontotermes sp aff erraticus, C. ligniperda, A. moschata et $P$. ulmi n'ont pas été observées pas ces auteurs. Ces résultats témoignent l'importance de la biodiversité des arthropodes ravageurs dans les parcelles de manioc au Tchad. Au Sénégal, Odontotermes sp aff erraticus a été rencontrée par Faye (2016). Cela montre l'intervention de la zone dans la diversité des espèces.

L'abondance de la cochenille farineuse varie en fonction des sites. Les sites 
de Faya et de Kobdogue abritent plus de cochenilles que ceux de Moya et Yaroungou. Ce résultat peut s'expliquer par le fait qu'à Faya et à Kobdogue, la végétation est très importante et aurait un effet sur l'abondance de cet insecte. La diversité végétale au sein d'un site offre une diversification des ressources alimentaires et un refuge pour les ravageurs et la faune auxiliaire (Nicholls et Altieri, 2012). L'abondance et la densité des populations d'un insecte donné sont contrôlées par de nombreux facteurs biotiques et abiotiques dans son écosystème, mais également par des facteurs intrinsèques à l'espèce (Sahraoui, 2012). La fluctuation des effectifs sous l'effet des facteurs physiques propres à chaque zone est importante pour l'interprétation des mécanismes de variation d'abondance du ravageur dans la nature et la mise en place des stratégies de lutte efficace.

La variété de manioc cultivée influe également sur les différents stades de développement de $P$. manihoti. En effet, les populations de l'espèce $P$. manihoti sont plus abondantes sur la variété Ngaloum nguegdje comparée à la variété Kangaba. Ces resultats revelent l'importance du choix de la variété dans la lutte contre la cochenille farineuse.

L'abondance des populations de l'espèce P.manihoti dans les sites de Faya et de Kobdogue s'expliquerait par le fait que la variété Ngaloum nguegdje est la plus cultivée dans ces zones. Cette variété serait plus sensible aux attaques de ce ravageur. L'espèce $P$. manihoti vit en colonies sur les parties jeunes et tendres des plants. Elle s'y nourrit en leur inoculant une toxine qui entraîne de sévères perturbations du développement des plants. Les pousses terminales prennent un aspect buissonnant, la croissance des plantes est ralentie, les entre-nœuds sont plus courts et les tiges se tordent. En cas d'infestations sévères, les plants dépérissent complètement en commençant par les sommités. Les attaques se développent surtout en saison sèche, et engendrent une chute de rendement des tubercules (Obame Minko, 2009).

Le nombre de populations de $M$. tanajoa varie en fonction des sites. La densité des populations de $M$. tanajoa est plus importante sur le site de kobdogue. Les populations de ce ravageur peuvent augmenter rapidement quand les températures augmentent dans les régions chaudes et tropicales (Bellotti, 2008). La zone tropicale offre des conditions favorables à $M$. tanajoa pendant la saison sèche lorsque les températures moyennes sont de 24 à $31^{\circ} \mathrm{C}$ et les valeurs d'humidité relative sont de 50 à $70 \%$ (Herrera et al., 2011). Au Congo où la présence de l'acarien vert se manifeste par des niveaux de densité variable. En effet, plusieurs variétés de manioc sont cultivées en milieu paysan mais le choix des variétés est fonction de plusieurs critères. Ces critères sont liés non seulement à des caractéristiques propres aux variétés mais aussi à des facteurs liés à l'environnement, aux contraintes de production et de commercialisation (Fagbemissi, 2001).

La population de l'acarien vert était plus importante sur la variété Ngaloum nguegdje. Cette variété serait plus attractive aux acariens et donc plus exposée aux attaques du ravageur. Selon Night et al. (2011), la gravité des attaques de l'acarien vert du manioc était plus élevée sur les cultivars locaux. Les attaques de l'acarien vert peuvent entraîner des pertes de rendement allant de 20 à $80 \%$ sur le manioc (Mahungu et al., 2014). L'espèce $M$. tanajoa préfère la face inférieure des jeunes feuilles émergentes et utilise son stylet pour pénétrer le tissu foliaire. Les fortes infestations provoquent la défoliation de la partie apicale du manioc et tuent souvent les bourgeons apicaux et latéraux (Bellotti, 2002). La densité de la population de l'acarien vert a été plus faible sur la variété Kangaba qui s'est montrée plus résistante. Les variétés résistantes jouent un rôle important dans 
l'amélioration de l'efficacité de la lutte biologique. Ces résultats confirment ceux de Fagbemissi et al. (2002), montrant des variétés résistantes, favorables à la lutte biologique contre M. tanajoa au Benin. La résistance variétale entraverait, retarderait ou supprimerait l'abondance de la population de l'acarien vert $\mathrm{du}$ manioc. Elle pourrait favoriser l'introduction des populations des ennemies naturelles efficaces qui pourraient lutter contre M. tanajoa (Bellotti et al., 2012). Le développement rapide des populations de M. tanajoa sur les jeunes feuilles laisse supposer qu'elles ont une qualité nutritionnelle supérieure. Le taux d'azote contenu dans les feuilles a une influence significative sur la fécondité et le développement des populations de M. tanajoa. L'apparition de nouvelles feuilles est importante après les premières pluies. Cette période est par conséquent celle à laquelle le développement des populations de M. tanajoa est plus rapide (Badegana et Yombo, 2002).

La densité des populations de la mouche blanche a été plus importante à Kobdogue et à Faya. Ces résultats s'expliquent en partie par le fait que nos échantillonnages ont été effectués pendant la période de forte chaleur dans ces sites (mars, avril et mai). Cette tendance est confirmée par les résultats de Ntawuruhunga et al. (2002) montrant que la principale période de propagation de la mouche blanche (saison sèche) se situe entre avril, mai et juin sur la culture du manioc au Nigeria. Selon N'zi et al. (2010), l'abondance de la population de $B$. tabaci est favorisée par la quantité d'acides aminés essentiels importante dans les feuilles offrant ainsi une meilleure qualité nutritionnelle vis-à-vis des insectes. La faible abondance des populations de la mouche blanche dans les sites de Moya et Yaroungou serait alors un atout pour le développement de la production du manioc dans ces zones.
La variété de manioc influe sur les infestations due à la mouche blanche. Ces résultats confirment ceux de Omongo et al. (2012). L'abondance des populations de la mouche blanche a été plus importante sur la variété Ngaloum nguegdje comparée à la variété Kangaba. La faible infestation de la variété Kangaba s'expliquerait par le fait qu'elle soit plus amère que la variété $N$ galoum nguegdje. En effet les variétés amères de manioc sont moins attractives pour les ravageurs (Cliff et al., 2011).

\section{Conclusion}

Cette étude avait pour objectifs d'identifier les arthropodes ravageurs associés à la culture du manioc au Tchad et d'étudier leurs distributions en fonction des sites et des variétés cultivées. Les résultats ont montré que les principaux arthropodes ravageurs du manioc sont $P$. manihoti, B. tabaci et $M$. tanajoa. L'espèce $P$. manihoti est plus abondante sur les deux variétés étudiées. Elle est suivie par B. tabaci et ensuite M. tanajoa. Outre ces principaux ravageurs, d'autres bioagresseurs appartenant aux ordres des orthoptères (Zonocerus variegatus), des isoptères (Odontotermes sp aff erraticus), des Hyménoptères (Camponotus ligniperda) des coléoptères (Aromia moschata) et des Arachnides (Panonychus ulmi) ont été aussi récoltées.

L'abondance des principaux ravageurs du manioc varie en fonction des variétés et des sites. La variété Kangaba est moins attractive pour les ravageurs contrairement à la variété Ngaloum nguegdje qui s'est révélée attirante pour les ravageurs. Les sites de Kobdogue et de Faya ont enregistré d'importantes populations de la cochenille farineuse, de l'acarien vert et de la mouche blanche. Ces sites sont dominés par la culture de la variété Ngaloum nguegdje plus sensible aux attaques de tous les ravageurs étudiès. Par contre, les ravageurs sont plus 
faibles sur les sites de Yaroungou et Moya. Ces derniers sont essentiellement occupés par la variété Kangaba.

Ces résultats méritent d'être approfondis pour une meilleure connaissance des paramètres de défense des différentes variétés cultivées dans la région face aux ravageurs. Il serait également important d'étudier l'incidence de ces ravageurs dans les périmètres agricoles. Ces résultats fournissent des informations intéressantes pour adopter des stratégies de gestion des arthropodes ravageurs au Tchad.

\section{CONFLIT D'INTERETS}

Les auteurs déclarent qu'il n'y a aucun conflit d'intérêts.

\section{CONTRIBUTIONS DES AUTEURS}

Mahamat-Saleh Abderahim : Définition des protocoles expérimentaux, collecte des données sur le terrain, nalyse statistique des données, rédaction du document; Mamadou Diatte : Définition des protocoles expérimentaux, Analyse statistique des données, Rédaction du document; Babacar Labou: Conduite des sorties de terrain, Rédaction du document; Gallo Sow : Rédaction du document; Karamoko Diarra: Coordination des activités, Définition des protocoles expérimentaux, Analyse statistique des données, Correction du document.

\section{REMERCIEMENTS}

Nous remercions profondément les personnes et les organismes qui sont intervenus lors de cette étude. Il s'agit particulièrement des producteurs de Maro (zone d'étude) et à toute l'Equipe production et Protection Intégrées en Agroécosystèmes horticoles 2PIA' de l'Université Cheikh Anta Diop pour leur appui technique.

\section{REFERENCES}

Agre AP, Kouchade S, Odjo T, Dansi M, Nzobadila B, Assogba P, Dansi A, Akoegninou A, Sanni A. 2015. Diversité et évaluation participative des cultivars $\mathrm{du}$ manioc (Manihot esculenta Crantz) au Centre Benin. Int. J. Biol. Chem. Sci., 9(1): 388-408.

DOI: http://dx.doi.org/10.4314/ijbcs.v9i1.33

Badegana AM, Yombo G. 2002. Influence de l'âge de la feuille sur les paramètres biologiques et les populations de l'acarien vert du manioc Mononychellus tanajoa Bondar (Acari : tetranychidae). Trop., 20 (3): 125-129.

Bellotti AC, Herrera CBV, Hyman G. 2012. Cassava Production and Pest Management: Present and Potential Threats in a Changing Environment. Trop. Plant Biol., 5(1): 39-72. DOI : 10.1007/s12042-011-9091-4

Bellotti AC. 2008. Cassava Pests and their Management. Encyclopedia of Entomology (2nd Edn), Capinera JL (ed). Springer: Dordrecht, The Netherlands.

Bellotti AC. 2002. Arthropod pests. In Cassava: Biology, Production and Utilization, Hillocks RJ, Thresh JM, Bellotti AC (eds). CABI Publishing: Wallingford, 209-235.

Cacai GHT, Ahanhanzo C, Dangou JS, Houedjissin SS, Agbangla C. 2012. Effets de différentes combinaisons hormonales sur l'organogenèse in vitro de quelques cultivars locaux et variétés améliorées de Manihot esculenta Crantz (manioc-Euphorbiaceae) cultivées au Bénin. Int. J. Biol. Chem. Sci., 6(4): 1593-1607.

DOI: http://dx.doi.org/10.4314/ijbcs.v6i4.19

Calvert LA, Thresh JM. 2002. The viruses and virus diseases of cassava. In Cassava: Biology, Production and Utilization, Hillocks J, Thresh JM, Bellotti AC (eds). 
CABI Publishing: Walling-ford, 237260.

Cliff J, Muquingue H, Nzwalo H, Bradbury JH. 2011. Konzo and continuing cyanide intoxication from cassava in Mozambique.Workshop on toxiconutritional neurodegenerations, September 2009, IPBO-Ugent, Belgium. Food Chem. Toxicol., 49 : 631-635.

Djinadou AKA, Olodo NI, Adjanohoun A. 2018. Evaluation du comportement de variétés améliorées de manioc riches en bêta-carotène au Sud du Bénin. Int. J. Biol. Chem. Sci., 12(2): 703-715. DOI: https://dx.doi.org/10.4314/ijbcs.v12i2.8

Fagbemissi RC, Coulibaly O, Hanna R, Endamana D. 2002. Adoption de variétés de manioc et efficacité durable de la lutte biologique contre l'acarien vert $\mathrm{du}$ manioc au Bénin. IITA, p.17.

Fagbemissi CR. 2001. Rôle des femmes rurales dans l'adoption des innovations technologiques en milieu rural. Cas des technologies de protection durable des cultures vivrières au Bénin. Mémoire de DEA, Université de Lomé, Lomé, p. 94.

FAO. 2012. Le manioc, une sécurité alimentaire d'avenir, 27 Avril 2012, Ndjamena, Rome. http://faostat.fao.org; consulté le 09/09/2016.

Faye A. 2016. Etude comparative de variétés de manioc (Manihot esculenta CRANTZ) selon leur sensibilité aux termites ravageurs des boutures dans le département de Tivaouane (Sénégal) et leurs capacités organogénétiques in vitro. Thèse de Doctorat $\mathrm{PhD}$, Université Cheikh Anta Dakar, Dakar, p. 102.

Herrera CBV, Hyman G, Bellotti A. 2011. Threats to cassava production: known and potential geographic distribution of four key biotic constraints. Food Sec, 3(3): 329-345.
IITA. 2000. Lutte contre les ravageurs du manioc. Centre de lutte biologique en Afrique. Cotonou, Bénin, p. 2.

Mahamat Saleh A. 2010. Résistance variétale à la Mosaïque africaine et à la cochenille farineuse (Phénacoccus manihoti) de onze clones de manioc du Nigeria. Mémoire de Master 2, UCAD, Dakar, p 35.

Mahungu NM, Tata Hangy KW ; Bidiaka SM, Frangoie A. 2014. Multiplication de matériel de plantation de manioc et gestion des maladies et ravageurs. Manuel de formation destiné aux agents de terrain. IITA/RDC, 24-34.

Nadjiam D, Diallo M, Mbaïguinam JMM, Guisse A. 2016. Pratiques paysannes de gestion des cultivars de manioc (Manihot esculenta Crantz) au Sud du Tchad. Int. J. Biol. Chem. Sci., 10(3): 1098-1113. DOI: http://dx.doi.org/10.4314/ijbcs.v10i3.16

Nicholls CI, Altieri MA. 2012. Plant biodiversity enhances bees and other insect pollinators in agroecosystems. Agro. Sust. Dev., 33(2): 257-274. DOI : 10.1007/s13593-012-0092-y

Night G, Asiimwe P, Gashaka G, Nkezabahizi D, Legg D, Okao-Okuja G, Obonyo R, Nyirahorana C, Mukakanyana C, Mukase F, Munyabarenzi I, Mutumwinka M. 2011. Occurrence and distribution of cassava pests and diseases in Rwanda. Agriculture, Ecosystems and Environment, 140 : $492-497$.

Ntawuruhunga P, Okuja O, Legg J, Albert Bembe A, Obambi M. 2002. Situation de la maladie pandémique virale de la mosaïque du manioc en République du Congo Rapport diagnostique d'enquête sur les maladies et les pestes de la culture du manioc en République du Congo, p. 37.

N'zi JC, Kouamé C, N'guetta ASP, Fondio L, Djidj AH, Sangare A. 2010. Évolution 
des populations de Bemisia tabaci Genn. selon les variétés de tomate (Solanum lycopersicum L.) au Centre de la Côte d'Ivoire. Sciences et Nature, 7(1) : 3140.

Obame Minko D. 2009. Influence des facteurs écologiques (température et hygrométrie) sur le développement de la cochenille farineuse du manioc (Phenacoccus manihoti Matile-Ferrero, Homoptera : Pseudococcidae). Trop. Tri., 27(1) : 21 25. URL : http:// www.tropicultura.org/text/v27n1.pdf

Omongo CA, Kawuki R, Bellotti AC, Alicai $\mathrm{T}$, Baguma Y, Maruthi MN, Bua A, Colvin J. 2012. African cassava whitefly, Bemisia tabaci, resistance in African and
South American cassava genotypes. $J$. Integr. Agric., 11: 327-336. DOI: 10.1016/s2095-3119(12)60017-3

Papy HB, Willy LT. 2012. Adoption des variétés améliorées de manioc en milieu paysan. Le Semeur du Kasaï. Rev. Pluri., 1 : 54-61.

Sahraoui H. 2012. Influence des pratiques agro-écologiques et de la protection phytosanitaire sur les communautés d'acariens Phytoseiidae (Acari : mesostigmata) dans les vergers agrumicoles tunisiens. Thèse de Doctorat, Institut National Agronomique de Tunisie, Montpellier Supagro, Montpellier, p. 187. 